TecnoLógicas

ISSN 0123-7799

ISSN-e 2256-5337

Vol. 20, No. 39

Mayo - agosto de 2017

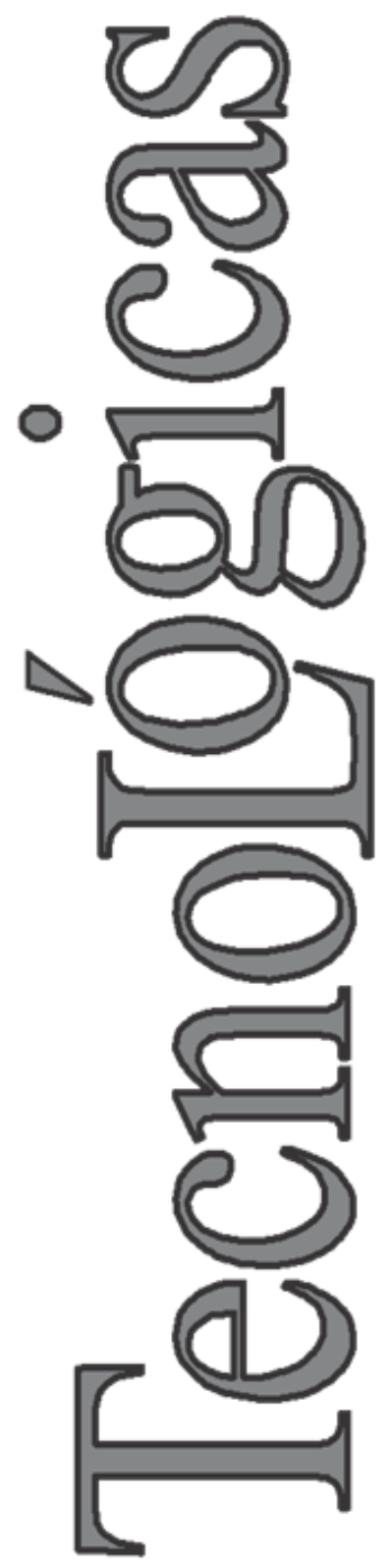

(C) Copyright 2015 por autores y Tecno Lógicas Este trabajo está licenciado bajo una Licencia Internacional Creative Commons Atribución (CC BY)

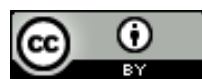

\section{Diseño y construcción de un sistema híbrido de calentamiento de aire por combustión de biomasa y radiación solar, utilizando PCM como fuente de almacenamiento térmico, para secado de yuca}

\section{Design and construction of a hybrid system of heating air by combustion of biomass and solar radiation, using phase change material (PCM) as a source of thermal storage, for cassava drying}

\author{
Ramiro Torres-Gallo, Pedro J. Miranda-Lugo ${ }^{2}$ \\ y Keimer A. Martínez-Padilla ${ }^{3}$
}

Recibido: 20 de noviembre de 2015,

Aceptado: 15 de marzo de 2017

Cómo citar / How to cite

R. Torres-Gallo, P.J. Miranda-Lugo y K.A. Martínez-Padilla, "Diseño y construcción de un sistema híbrido de calentamiento de aire por combustión de biomasa y radiación solar, utilizando PCM como fuente de almacenamiento térmico, para secado de yuca.”, TecnoLógicas, vol. 20, no. 39, 2017.

1 MSc en Ciencias Agroalimentarias, Ingeniero Químico, Facultad de Ingeniería, Departamento de Ingeniería Agroindustrial, Universidad del Atlántico, Barranquilla-Colombia, ramirotorres@mail.uniatlantico.edu.co

2 Estudiante de Ingeniería Mecánica, Facultad de Ingeniería, Departamento de Ingeniería Mecánica, Universidad de Córdoba, Montería-Colombia,pjose1028@gmail.com

3 Estudiante de Ingeniería Mecánica, Facultad de Ingeniería, Departamento de Ingeniería Mecánica, Universidad de Córdoba, Montería-Colombia, kealmapa@gmail.com 


\section{Resumen}

Este estudio consistió en el diseño, la construcción y la validación de un sistema híbrido de calentamiento de aire por combustión de biomasa y radiación solar, utilizando material de cambio de fase (PCM) como fuente de almacenamiento térmico, para el secado de yuca, a pequeña escala. El sistema híbrido consta de un ventilador centrífugo, dos colectores solares, un quemador de combustible sólido (cascarilla de arroz) y un secador de bandejas. La validación del sistema se realizó secando yuca. El PCM permitió seguir el proceso de secado, incluso cuando la radiación solar estuvo por debajo de 116,22 $\pm 31,94 \mathrm{~W} / \mathrm{m} 2$, logrando mantener temperaturas de aire de secado en los dos colectores solares a $46 \pm 4,29^{\circ} \mathrm{C}$ y $51 \pm 4,08^{\circ} \mathrm{C}$ durante 45 min adicionales. El tiempo de secado fue de $10 \mathrm{~h}$ y $45 \mathrm{~min}$, la eficiencia de los colectores solares fue $43,91 \%$ y la del quemador de cascarilla de arroz de $36,72 \%$.

\section{Palabras clave}

Secado, yuca, PCM, tiempo de secado, híbrido, calentamiento.

\section{Abstract}

This study consisted of designing, building and validation a hybrid system of heating air by combustion of biomass and solar radiation, using phase change material (PCM) as a thermal storage source, for cassava drying, a small scale. The dryer consists of a centrifugal fan, two solar collectors, a fuel burner solid (rice husk) and a tray dryer. System validation was performed drying up Yucca. The PCM allowed to follow the drying process, even when the solar radiation was below 116,22 $\pm 31,94 \mathrm{~W} / \mathrm{m} 2$, being able to maintain drying air temperatures in the two solar collectors at $46 \pm 4,29^{\circ} \mathrm{C}$ and $51 \pm$ $4.08^{\circ} \mathrm{C}$ for an additional $45 \mathrm{~min}$. The drying time was $10 \mathrm{~h}$ and $45 \mathrm{~min}$, the efficiency of the solar collectors was $43.91 \%$ and the rice husk burner of $36.72 \%$.

\section{Keywords}

Drying, cassava, PCM, hybrid, heating. 


\section{INTRODUCCIÓN}

El secado o deshidratación se usa como técnica de preservación de alimentos, donde se le reduce su humedad, hasta un valor aceptablemente bajo. Es un método que permite alargar la vida útil de los alimentos, pues los microorganismos que causan los cambios químicos en los alimentos, no pueden crecer y desarrollarse en ausencia de agua. Los microorganismos dejan de ser activos cuando el contenido de agua se reduce por debajo del 10\% en peso [1], [2]. Según Ibarz y Barbosa-Cánovas [2] los términos de secado y deshidratación, aunque se utilizan como sinónimos, técnicamente son diferentes ya que un alimento se considera deshidratado cuando tiene menos de 2,5\% de agua, sin embargo, seco puede contener más de $2,5 \%$.

El diseño de secadores solares y su desarrollo, es una prioridad de la industria de alimentos, porque la energía solar es considerada hoy en el mundo como una de las de mayor proyección, debido a los conocidos problemas de agotamiento de las fuentes fósiles de combustible, y su correlación con la problemática de las emisiones de gases de efecto invernadero, además del alto consumo energético en los procesos de secado convencionales [3].

La técnica de secado empleada para la deshidratación frutas, granos, vegetales, carnes y pescados ha sido utilizadas por cientos de años de forma poco tecnificada, y el método más conocido es el secado solar abierto o secador solar natural. Sin embargo, presenta limitaciones como falta de control sobre el proceso de secado, que puede ocasionar excesivo secado, pérdida de granos en germinación y cambios nutricionales; falta de uniformidad del secado, contaminación por hongos, bacterias, roedores, pájaros o insectos, las condiciones del clima y debido a que la velocidad del secado es lenta y los productos no pueden ser secados durante la noche deben ser recogidos y redistribuidos, ocasionando pérdida de calidad y precio del producto [2]. Debido a ello, se han desarrollado secaderos solares, todos basados en la utilización de colectores de energía solar (Fig. 1) donde la energía proveniente del Sol es utilizada para calentar el aire para el secado del alimento [2], [3]

Los colectores de energía más utilizado en secado de productos agrícolas son los de placa plana, pues proporcionan temperaras hasta $100^{\circ} \mathrm{C}$ por arriba de la temperatura ambiente [4]. Aunque el aprovechamiento energía solar solo en las horas de sol del día es una limitación a tener en cuenta cuando se plantea su utilización en una escala de importancia, sin embargo, los sistemas de acumulación presentan una alternativa para este problema [5].

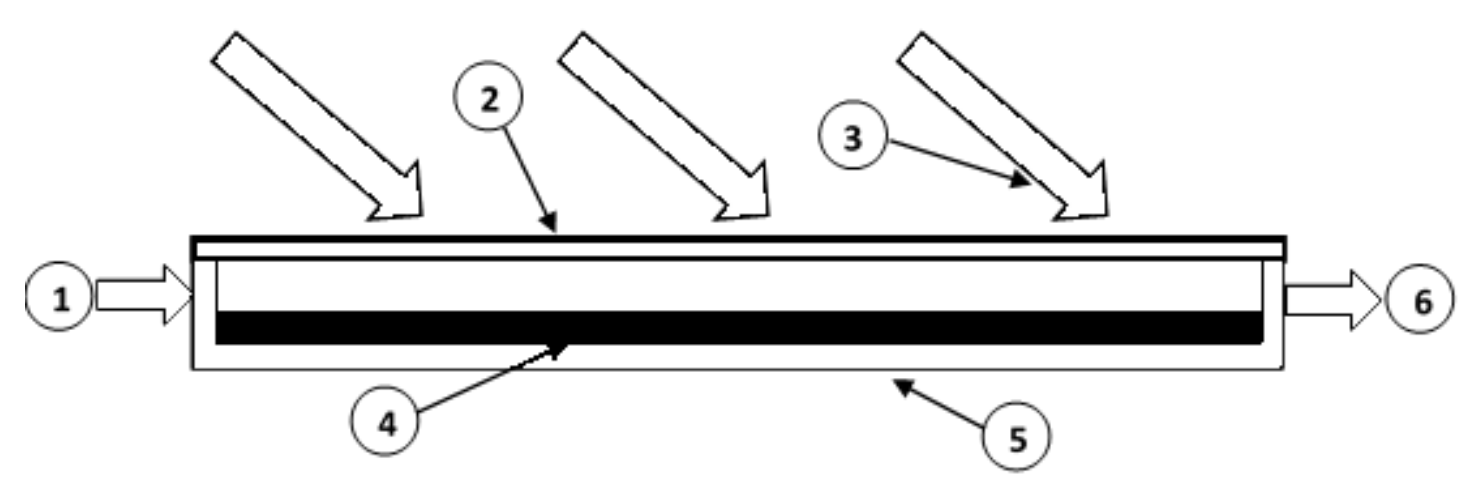

Fig. 1. Colector solar. (1) Entrada de aire frío, (2) Cubierta translúcida, (3) Radiación incidente, (4) Placa absorbedora, (5) Carcasa aislada (6) Salida aire caliente. Fuente Autores. 
Uno de los sistemas de acumulación que han despertado mucho interés en investigaciones en el campo de la energía solar durante los últimos años son los materiales que presentan cambio de fase (PCM, por sus siglas en inglés, Phase Change Materials). Lo interesante de estos sistemas es el hecho de que una vez el material empleado llega a la temperatura de cambio de fase, este sigue acumulando energía en forma de calor latente, a temperatura constante, hasta que se produce todo el cambio de estado y al caer la fuente de energía solar, el material comienza a ceder el calor acumulado, retornando a su estado de menor contenido energético, logrando de esta manera extender el proceso de secado [6].

Actualmente se tiene la tendencia a utilizar sistemas híbridos que permiten tener mayor control del proceso y mayores velocidades de secado debido al uso de fuentes de energía térmica, complementarias a la radiación solar [7], [8]. Se han reportado estudios sobre modelado dinámico, diseño y construcción de secadores para productos agrícolas, evaluando su cinética de secado y comparando su eficiencia con el secado solar natural. Condorí et al. [9], diseñaron y construyeron dos secadores solares del tipo indirecto con convección de aire forzada para una planta de deshidratado de productos frutihortícolas.

Acosta et al. [10] plantearon un modelo de fluidos y termotransferencia que permite predecir y mejorar el funcionamiento de un secador solar, asistido con gas o electricidad, las 24 horas del día. Fonseca et al. [11] evaluaron un sistema combinado de lecho fijo y secado en silos con aire a temperatura ambiente, para el secado del café; y [12] diseñaron y construyeron un secador solar híbrido para producción continua de pimentones deshidratado, utilizando conjuntamente un quemador de leña y un banco de colectores solares para calentar el aire.

Por su parte [13] diseñaron un prototipo de secador solar indirecto con un que- mador de biomasa, para el uso de agricultores rurales para secar bayas de pimienta en 16 horas. Yunus et al. [14] diseñaron una unidad térmica flexible para suministrar aire seco caliente para secar los alimentos. Tarigan y Tekasakul [15] desarrollaron un secador solar integrado para cacahuetes, con un quemador de biomasa, diseñado con capacidad 60 a $65 \mathrm{~kg}$ de producto procesado con una eficiencia del componente solar del 23\% y del quemador del 40\%. Prasad et al. [16] utilizaron un sistema híbrido de energía solar y biomasa para secador la planta medicinal Cúrcuma Long L, con flujo continuo de aire a temperatura entre 55 y $60^{\circ} \mathrm{C}$ obteniendo un tiempo de 1,5 días, mientras que el secado al sol abierto tomó 11 días para secar los rizomas. Cakmak e Yıldız [17] determinaron la cinética de secado de uvas en secador solar con colector solar integrado, utilizando acetato de sodio trihidratado como PCM. Por su parte [18] estudiaron el efecto de la utilización de la cera de parafina como medio de almacenamiento de energía térmica para la cinética de secado de productos alimenticios.

El objetivo de esta investigación fue diseñar, construir y validar el funcionamiento de un sistema híbrido de calentamiento de aire por combustión de biomasa y radiación solar que utiliza parafina (PCM) como fuente de almacenamiento de energía térmica, para el de yuca a escala piloto.

\section{METOdOLOGíA}

La investigación se realizó en la ciudad de Montería, donde los valores de radiación solar fueron tomados aleatoriamente de un conjunto de mediciones de radiación solar en la ciudad de Montería, entre los meses de enero y mayo del año 2015.

Se procedió en primera instancia a determinar la cantidad de humedad de la yuca a secar necesaria en el diseño de los equipos de secado. Se optó por diseñar un secador híbrido (Fig. 2), donde el calenta- 
miento del aire se realizaría utilizando un colector solar con parafina con PMC, para dar mayor aprovechamiento de la radiación solar y un quemador de biomasa (cascarilla de arroz) para aprovechar los desechos de la industria arrocera, en lugar de los combustibles fósiles comúnmente utilizados, dado las ventajas medioambientales de primer orden, como son: Disminución de las emisiones de azufre y de partículas, ciclo neutro de $\mathrm{CO} 2$, sin contribución al efecto invernadero, reducción del mantenimiento y de los peligros derivados del escape de gases tóxicos y combustibles, reducción de riesgos de incendios forestales y de plagas de insectos y aprovechamiento de residuos agrícolas, evitando su quema en el terreno [19].

\subsection{Caracterización de la humedad de la yuca}

Se determinó el porcentaje de humedad inicial de la Yuca, según el método AOAC 925.10 , por secado en estufa a $105^{\circ} \mathrm{C}$ durante un tiempo de 8 horas [20]. Además, se determinó la curva de secado a $70{ }^{\circ} \mathrm{C}$ utilizando la metodología empleada por [21], en cual consistió en secar rodajas de yuca con aire a $70 \pm 1{ }^{\circ} \mathrm{C}$ y velocidad $1 \mathrm{~m} / \mathrm{s}$. Durante el proceso de secado las muestras fueron pesadas a intervalos de 5 minutos durante la primera hora y luego, cada 15 minutos hasta obtener peso un constante.

\subsubsection{Simulación térmica del fenómeno de transferencia de calor en el colector solar}

Se realizó una simulación del proceso térmico en un colector solar de placa plana, utilizando el software Matlab ${ }^{\circledR}$, para determinar el comportamiento de las temperaturas de los componentes del colector (placa absorbedora, cubierta de vidrio, PCM) y aire en el colector, el número de latas llenas de parafina y el tiempo de secado necesarias para llevar a cabo el calentamiento del aire (Fig.3). Para determinar el número adecuado de latas llenas de parafina a utilizar en el colector solar, se utilizó como criterio de diseño: el menor tiempo en llegar la temperatura del aire al estado estacionario, sin sobrepasar las 2 horas de proceso, que se cumpliera con un régimen turbulento del aire de secado, para obtener mayores coeficientes de transferencia de calor y que la temperatura del PCM no sobrepasara su temperatura su fusión $\left(48^{\circ} \mathrm{C}\right)$.

En el modelo de simulación se consideró que el aumento de la temperatura a través del tiempo se dio en el siguiente orden: placa absorbedora $(\mathrm{P})>$ cubierta de vidrio $>$ PCM (parafina) > aire, lo que determinó la dirección del flujo de transferencia de calor y solo se tuvieron en cuenta la transferencia de calor por convección y radiación, de acuerdo con las ecuaciones de balance de energía para el aire (1), el PCM (2), el vidrio (3) y la placa absorbedora (4).

$$
\begin{aligned}
\rho_{1} q_{\text {aire }, 1} C_{p} T_{1}(t) & +h_{P, A} A_{P, A}\left(T_{P}(t)-T_{2}(t)\right)+h_{v, A} A_{v, A}\left(T_{v}(t)-T_{2}(t)\right) \\
& +h_{P C M, A} A_{P C M, A}\left(T_{P C M}(t)-T_{2}(t)\right)-\rho_{2} q_{\text {aire }, 2} C_{p} T_{2}(t)=\rho_{A} v_{A} C_{v, A} \frac{d T_{2}(t)}{d t} \\
\alpha_{\text {aluminio }} A_{P C M}{ }^{(r)} I_{\text {rad }}+F_{(P, P C M)} \varepsilon_{P} \sigma A_{P, A}\left(T_{P}(t)^{4}-T_{P C M}(t)^{4}\right)+F_{(v, P C M)} \varepsilon_{v} \sigma A_{V}\left(T_{v}(t)^{4}-T_{p c m}(t)^{4}\right) & d T_{P C M}(t) \\
& \left.-h_{P C M, A} A_{P C M, A}\left(T_{P C M}(t)-T_{2}(t)\right)=\rho_{P C M} v_{P C M} C_{v, P C M} \frac{d t}{F_{(P, v)} \varepsilon_{P} \sigma A_{P}\left(T_{P}(t)^{4}\right.}-T_{v}(t)^{4}\right)-F_{(v, P C M)} \varepsilon_{v} \sigma A_{v}\left(T_{v}(t)^{4}-T_{p c m}(t)^{4}\right)-h_{v, A} A_{v, A}\left(T_{v}(t)-T_{2}(t)\right) \\
& =\rho_{v} v_{v} C_{v, v i d r i o} \frac{d T_{v}(t)}{d t}
\end{aligned}
$$




$$
\begin{gathered}
\alpha_{\text {aluminio }} A_{P}{ }^{(r)}\left(I_{\text {rad }}\right)-F_{(P, v)} \varepsilon_{P} \sigma A_{P}\left(T_{P}(t)^{4}-T_{v}(t)^{4}\right)-F_{(P, P C M)} \varepsilon_{P} \sigma A_{P}\left(T_{P}(t)^{4}-T_{P C M}(t)^{4}\right) \\
-h_{P, A} A_{P, A}\left(T_{P}(t)-T_{2}(t)\right)=\rho_{\text {aluminio }} v_{p} C_{v, \text { placa }} \frac{d T_{P}(t)}{d t}
\end{gathered}
$$

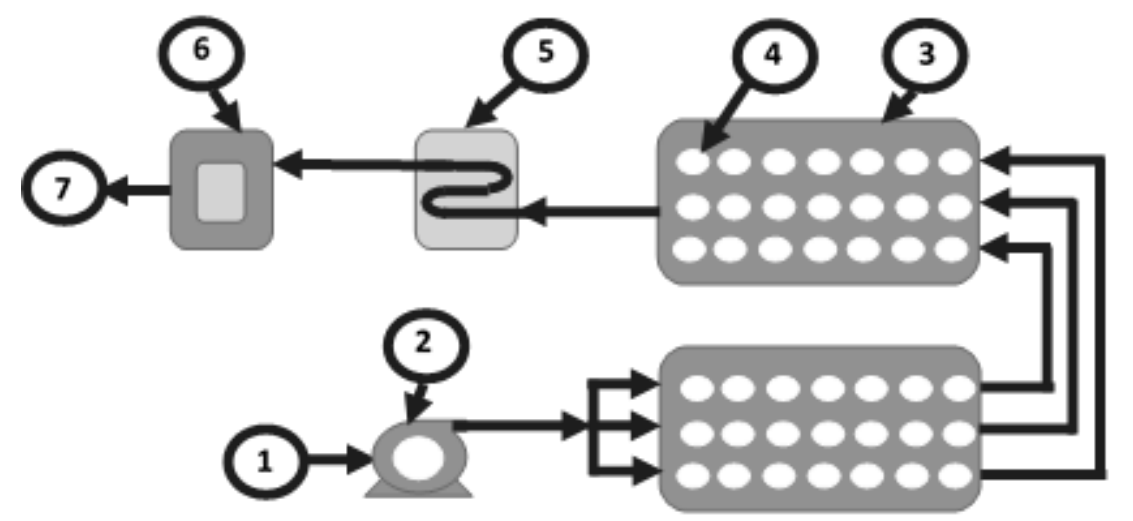

Fig. 2. Diagrama flujo de secador híbrido. Organización: (1) Entrada de aire al sistema, (2) Soplador, (3) Colectores, (4) tubos conteniendo PMC, (5) Quemador de Biomasa, (6) secador y (7) Salida de aire del sistema. Fuente: Autores.

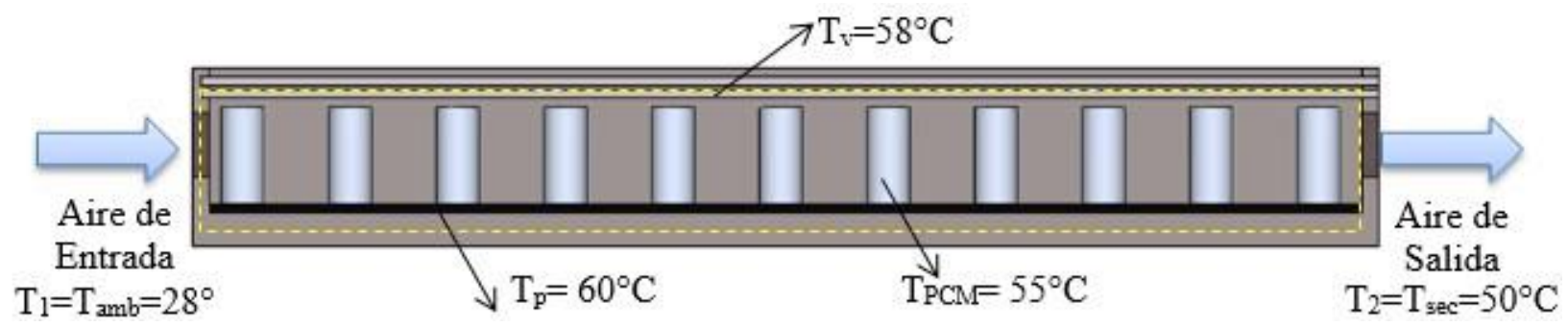

Fig. 3. El volumen de control considerado en la simulación térmica del fenómeno de transferencia de calor en el colector solar, donde $T_{V}$ es la temperatura de placa de vidrio, $T_{P}$ es temperatura de placa absorbedora, TPCM es la temperatura de PCM (parafina) $\mathrm{T}_{1} \mathrm{y} \mathrm{T}_{2}$ son las temperaturas del aire a la entrada y salida de colectores reactivamente. Fuente: Autores.

También, se consideraron los factores de forma de radiación entre los componentes, para ello se asumió un corte transversal a lo largo del colector solar y se utilizaron las ecuaciones para configuraciones en dos dimensiones. Se asumió que la radiación solar incide sobre la parte superior y la mitad del haz de tubo, obteniendo de esta manera el área de transferencia de calor entre la parafina y el aire.

\subsection{Diseño, construcción y validación siste- ma híbrido de calentamiento}

El diseño del colector solar para calentamiento de aire se realizó a partir de la energía requerida para secar $5 \mathrm{~kg}$ de yuca, empleando latas de aluminio de $330 \mathrm{~cm}^{3}$ rellenas de parafina blanca, con el fin de tener un sistema de almacenamiento de energía térmica que permitiera aumentar el rendimiento de los colectores. La parafina se escogió como PCM, teniendo en cuenta sus propiedades térmicas, fisicoquímicas, buena estabilidad y aspectos económi$\cos$ [22], [23].

Se diseñó un quemador de biomasa con intercambiador de calor, el combustible sólido escogido fue la cascarilla de arroz como alternativa de aprovechamiento integral [24]. Se determinó el área de intercambio de calor para mantener la temperatura de secado a $70^{\circ} \mathrm{C}$ y se calculó el flujo de los gases en la chimenea y la dimensión de la misma. Se consideró la construcción de un sistema de adquisición de datos las medidas de temperaturas en los colectores y el secador, así como la humedad relativa a la entrada y salida del secador. 
El sistema se validó con el secado de 5 $\mathrm{kg}$ de yuca picada en trozos distribuidos en un área de $0,15 \mathrm{~m}^{2}$, hasta retirar el $65 \%$ de su humedad inicial. Se midieron las temperaturas a la entrada y salida de los colectores, en la salida del quemador de biomasa y a la salida del secador. Se determinó la humedad el producto a través del tiempo, su humedad final y el tiempo de sacado. Los ensayos se realizaron por triplicado. La intensidad solar durante el ensayo se evaluó a través del medidor TES 1333 Solor power meter ${ }^{\circledR}$.

\section{RESULTADOS Y DISCUSIÓN}

\subsection{Porcentaje de humedad inicial por me- dio de una estufa de flujo estacionario en el laboratorio}

El porcentaje de humedad inicial de la yuca fue de $61,88 \pm 1,03 \%$ y el tiempo promedio de secado, para llevar la yuca hasta un porcentaje de humedad del $12 \%$ con una temperatura de secado de $70^{\circ} \mathrm{C}$, fue de $16 \mathrm{~h}$ y $11 \mathrm{~min} \pm 18 \mathrm{~min}$. La curva de tiempo de secado a $70{ }^{\circ} \mathrm{C}$ evidenció un periodo de velocidad de secado constante (rapidez de evaporación constante) hasta las $5 \mathrm{~h}$, donde el contenido de humedad crítico (Xc) fue de 0,29 g de h/g de sólido seco (SS). También evidenció un periodo de velocidad de secado decreciente (el cual se considera como un secado superficial no saturado) donde la velocidad de evaporación del agua disminuyó hasta un valor asintótico de $0,12 \mathrm{~g}$ de h/g de SS desde las 15 h, lográndose un secado homogéneo. En este periodo se considera que la película superficial del líquido se ha evaporado completamente (Fig. 4).

\subsection{Diseño y construcción del secador híbrido}

El número óptimo de latas rellenas de parafinas, calculados en la simulación, fue de 50 en cada colector, el cual que se de- terminó teniendo en cuenta como criterio de diseños esperados en la metodología: el menor tiempo en llegar la temperatura del aire al estado estacionario (5800 s), proceso con un régimen turbulento en flujo de aire de secado (Reynolds $=7,98 \times 104$ ) una temperatura del aire en colector de $46,09{ }^{\circ} \mathrm{C}$ (Fig. 5).

En la construcción del sistema híbrido se seleccionó un ventilador centrífugo de $559.27 \mathrm{~W}$ que proporcionara aire de secado con una eficiencia máxima $76 \%$, a una velocidad de giro de $920 \mathrm{rpm}$, en un rango de presión estática entre $0-150 \mathrm{~Pa}$ y un nivel de ruido menor a $49 \mathrm{~dB}$. El ventilador necesitó de un motor YDK 300-6D ® con conexión a $220 \mathrm{~V}, 3.1$ A y frecuencia de 50 $\mathrm{Hz}$ para cumplir con un flujo de aire máximo en el sistema de $3200 \mathrm{~m} 3 \mathrm{~h}-1$.

El ventilador fue acoplado, por medio de ductos de acero galvanizado de $7,62 \mathrm{~cm}$ de diámetro, a dos colectores conectado en serie a través de una tubería de PVC de 3 $7,62 \mathrm{~cm}$. Cada colector se construyó con los siguientes componentes: una caja de madera de roble pintados de negro con un espesor de $2 \mathrm{~cm}$ y un área de diseño de 1,12 $\mathrm{m} 2$, una placa absorbedora de aluminio de $0.7 \mathrm{~mm}$ de espesor de color negro (para que absorbiera la mayor cantidad de radiación solar), una lámina de poli-estireno expandido de $2 \mathrm{~cm}$ de espesor como aislante del sistema del ambiente y una cubierta de vidrio transparente de $77 \times 137.1 \mathrm{~mm}$ y 4 $\mathrm{mm}$ de espesor.

En el interior de cada uno de los colectores solares se ubicaron las 50 latas de aluminio de $330 \mathrm{~cm} 3$ rellenas de parafina blanca, dispuestas en forma escalonada, donde regímenes de flujo turbulento (Reynolds $>1 \mathrm{x} 104$ ), permitiendo mayor intercambio de calor. Las latas con parafina permitieron almacenar de energía térmica proveniente de la radiación para mantener la temperatura del aire de secado en 52 $\pm 3.46{ }^{\circ} \mathrm{C}$, cuando la radiación solar no fue suficiente, con una eficiencia de los colectores de $43,91 \%$ (Fig. 6). 
Diseño y construcción de un sistema híbrido de calentamiento de aire por combustión de biomasa y radiación solar, utilizando PCM como fuente de almacenamiento térmico, para secado de yuca

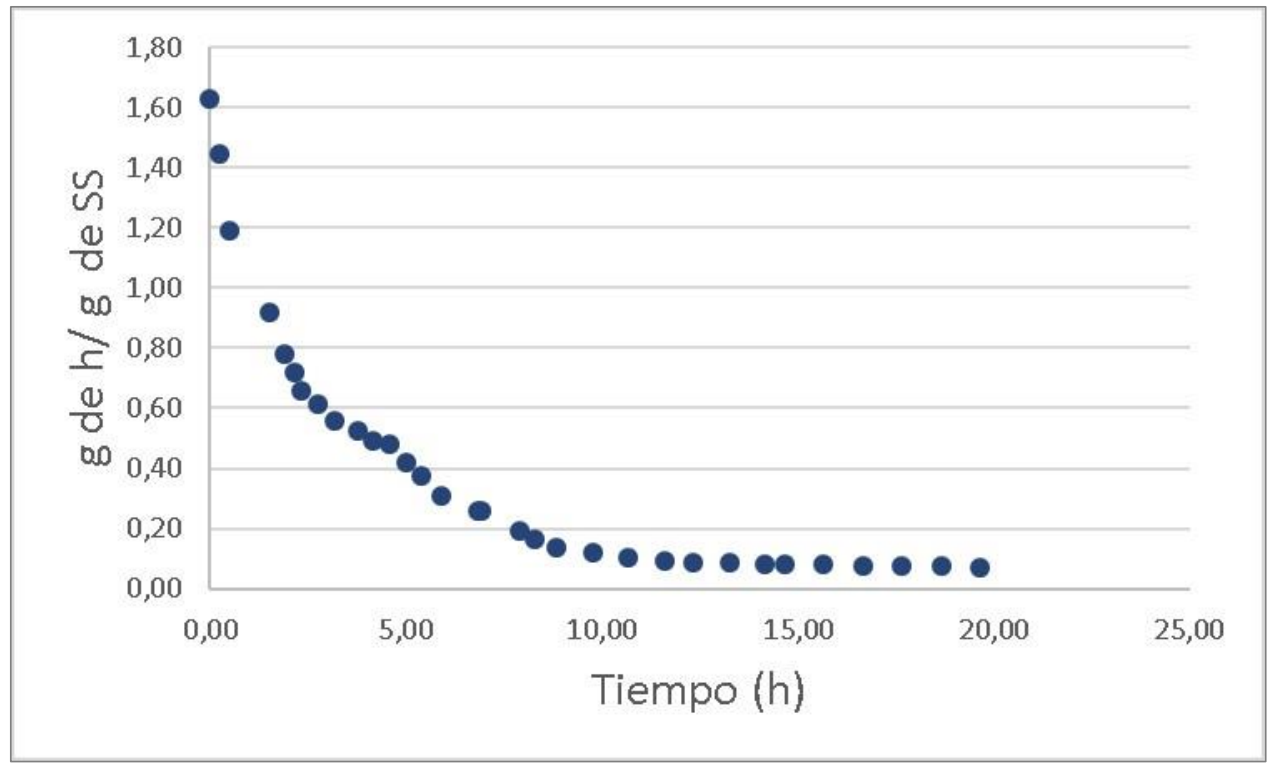

Fig. 4. Curva de secado a temperatura de $70{ }^{\circ} \mathrm{C}$ expresada en g de humedad (h)/ g de sólido seco (SS) vs Tiempo en h. Fuente: Autores.

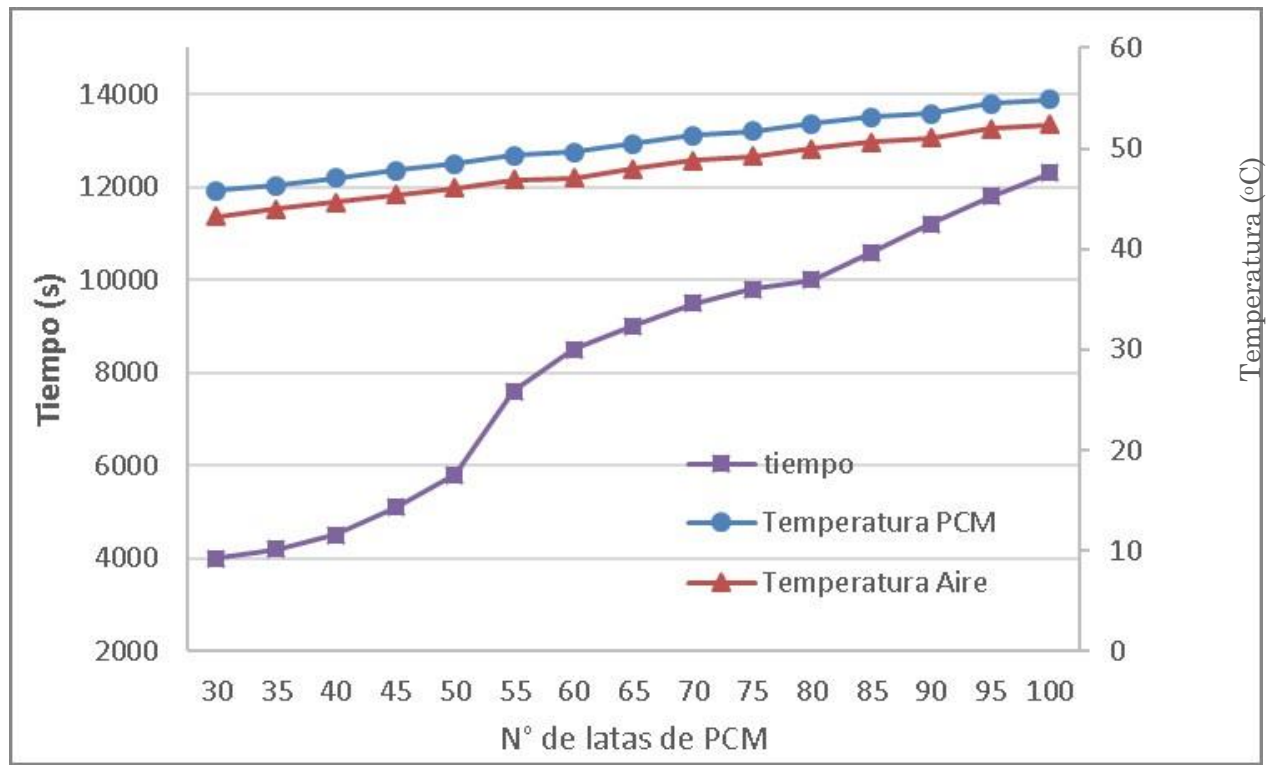

Fig. 5. Gráfico del tiempo en que se alcanzan las temperaturas del PCM (parafina) y del aire en función del número de latas que contiene PCM, obtenidos por simulación del proceso térmico. Fuente: Autores. 


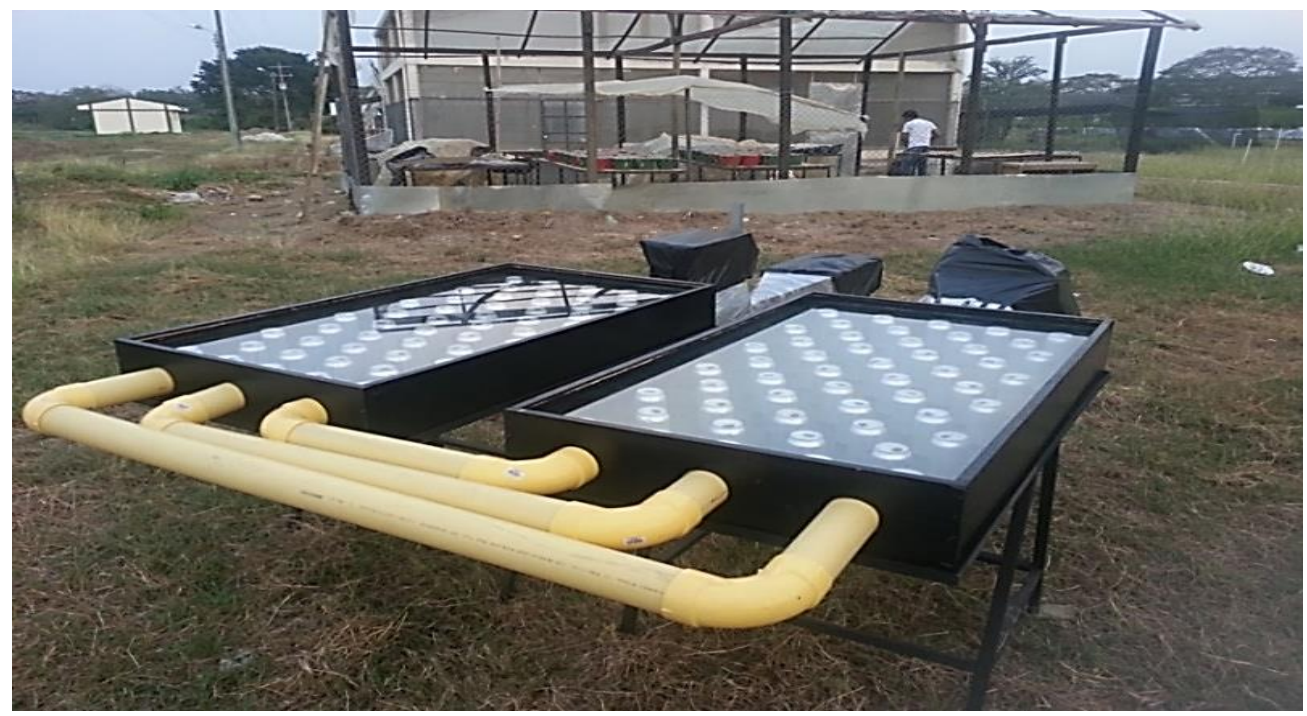

Fig. 6. Fotografía de los colectores solares. Fuente: Autores

El colector fue acoplado a un quemador de biomasa en uno de los dos extremos del intercambiador de calor que se encuentra en su interior (Fig. 7), el cual se diseñó con un área de intercambio de calor de 0,07 m2 y se construyó con tubo de cobre de $2,54 \mathrm{~cm}$ de diámetro y $2.81 \mathrm{~m}$ de longitud en forma de serpentín. El quemador fue construido de acero galvanizado, con una compuerta con ventana de vidrio que permite monitorear el estado de la combustión, una tolva con capacidad de $900 \mathrm{~g}$ de cascarilla de arroz, una malla de $0,90 \mathrm{~mm}$ para filtrar los sólidos producto de la combustión desechándolos en el fondo del quemador, una compuerta auxiliar para limpieza y una chimenea de 50 × $50 \mathrm{~mm}$ de sección transversal con altura de $325 \mathrm{~mm}$.

El otro extremo del intercambiador de calor fue acoplado al secador, el cual fue construido de láminas de acero galvanizado en forma de túnel (Fig.8), con $35 \mathrm{~cm}$ de ancho, 20,2 cm de alto y $55 \mathrm{~cm}$ de largo. Cada extremo del secador se diseñó en forma de difusor para que permitieran la expansión del aire de secado de forma gradual en su interior. El secador fue provisto de una malla de 5x30x $50 \mathrm{~cm}$ con el objetivo que el aire pase a través de todo el alimento logrando un secado homogéneo.
Se construyó e instaló el sistema de adquisición de datos para registrar las temperaturas en los colectores y el secador, así como la humedad relativa a la entrada y salida del secador. El sistema híbrido se puede observar en a Fig. 9.

\subsection{Operación y validación del secador híbrido}

El tiempo de secado de $5 \mathrm{~kg}$ de yuca con una humedad inicial del $62 \pm 1.03 \%$, picada en trozos distribuidos en un área de 0,15 $\mathrm{m} 2$, hasta $12 \%$ de su humedad final fue de 16 horas y 45 minutos, siendo 1 hora 33 min mayor al obtenido en el laboratorio a una temperatura de secado de $70^{\circ} \mathrm{C}$. El consumo cascarilla de arroz fue de $4,5 \mathrm{~kg}$ de cascarilla de arroz/ kg de yuca húmeda.

El aire de secado en el primer colector alcanzó una temperatura de $46 \pm 4.73^{\circ} \mathrm{C}$ y en el segundo colector de $51 \pm 4,08^{\circ} \mathrm{C}$. El PCM permitió seguir el proceso de secado, incluso cuando la radiación solar estuvo por debajo de $116,22 \pm 31,94 \mathrm{~W} / \mathrm{m} 2$, logrando mantener temperaturas de aire de secado en los dos colectores solares a $46 \pm 4$, $29^{\circ} \mathrm{C}$ y $51 \pm 4,08^{\circ} \mathrm{C}$ durante $45 \mathrm{~min}$ adicionales. El quemador de biomasa permitó incrementar la temperatura del aire precalentado en los colectores $6^{\circ} \mathrm{C}$ en promedio, 
lográndose una eficiencia térmica en el quemador del $36,72 \%$, manteniendo la temperatura del aire al interior del secador en $57 \pm 11,55^{\circ} \mathrm{C}$, a una velocidad de secado de $2 \mathrm{~m} / \mathrm{s}$.

La curva de tiempo de secado (Fig. 10) presentó un periodo de velocidad de secado constante hasta las $14 \mathrm{~h}$, donde se alcanzó una humedad de $0,28 \mathrm{~g}$ de $\mathrm{h} / \mathrm{g}$ de SS, apenas sobrepasando la humedad critica (Xc). Comparada con la curva de secado de laboratorio, a temperatura de secado de $70{ }^{\circ} \mathrm{C}$, presentó una diferencia de 9 horas. El periodo de velocidad de secado decreciente se presentó hasta las 16 horas y 45 minu- tos, cuando se alcanza la humedad $0,138 \mathrm{~g}$ de h/g de SS (12\% de humedad), 1 hora 45 minutos más que el comportamiento obtenido en el laboratorio. Este comportamiento concuerda con el reportado por [25], para Plátano, banano, yuca y mango, en un secador solar por convección natural; con lo reportado por [26] para secado de malva judía y hojas de menta en un secador solar doméstico. También concuerda con el reportado por [27], para secado de yuca, con secador de bandeja; y con lo reportado por [28], para secado de patatas, aunque con un menor tiempo de secado.
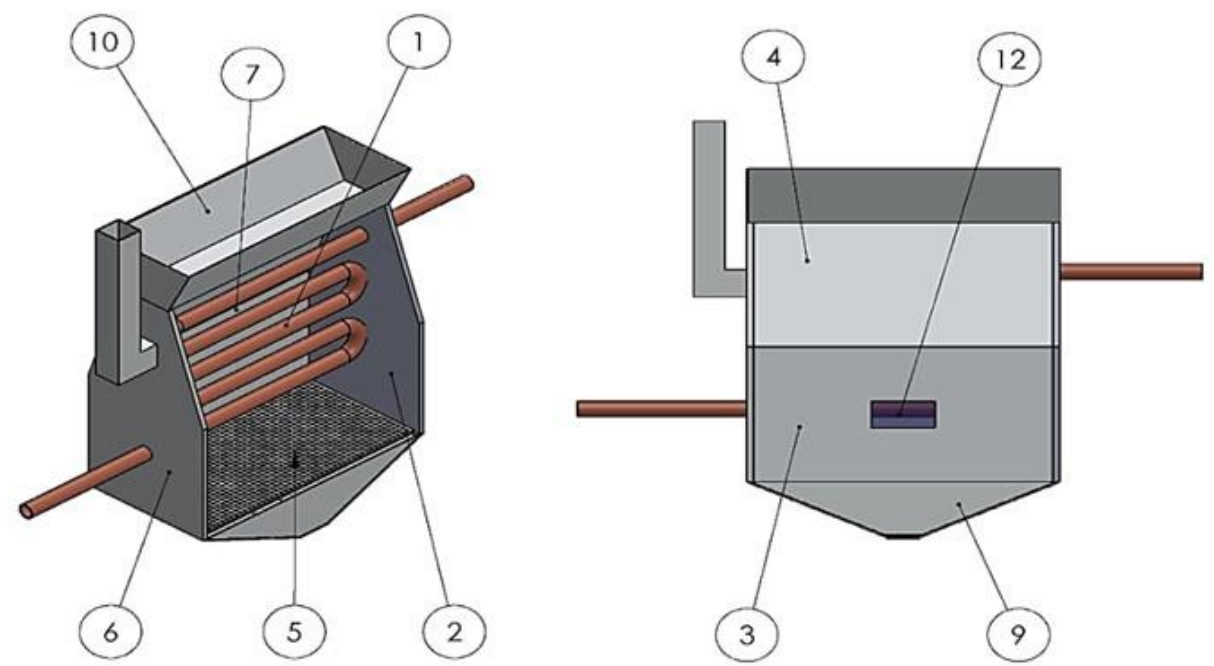

Fig. 7. Esquema quemador de biomasa. (1) Tubo del intercambiador, (2) Pared frontal interna, (3) Pared lateral, (4) Pared lateral inclinada, (5) Parrilla, (6) Soporte quemador, (7) Tolva, (8) Vidrio quemador. Fuente: Autores.

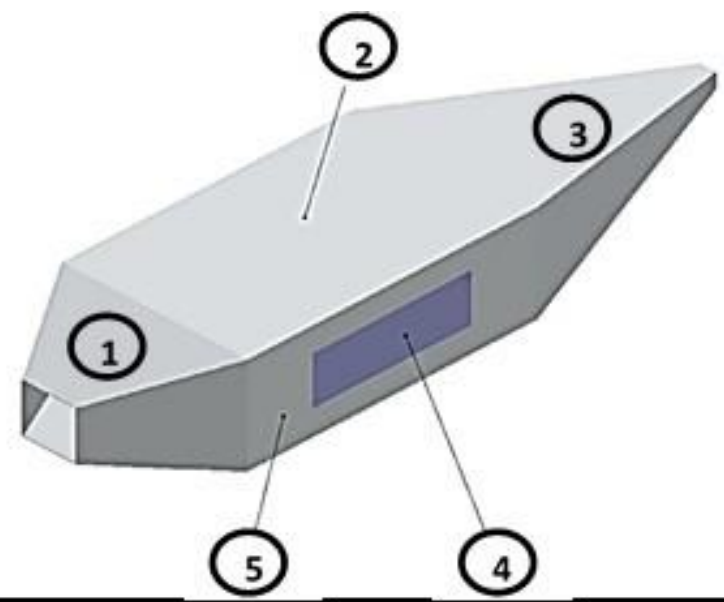

Fig. 8. Esquema del secador. (1) Tobera de salida, (2) Cuerpo del secador, pared superior, (3) Tobera de entrada, (4) Vidrio, (5) Tapa. Fuente: Autores. 


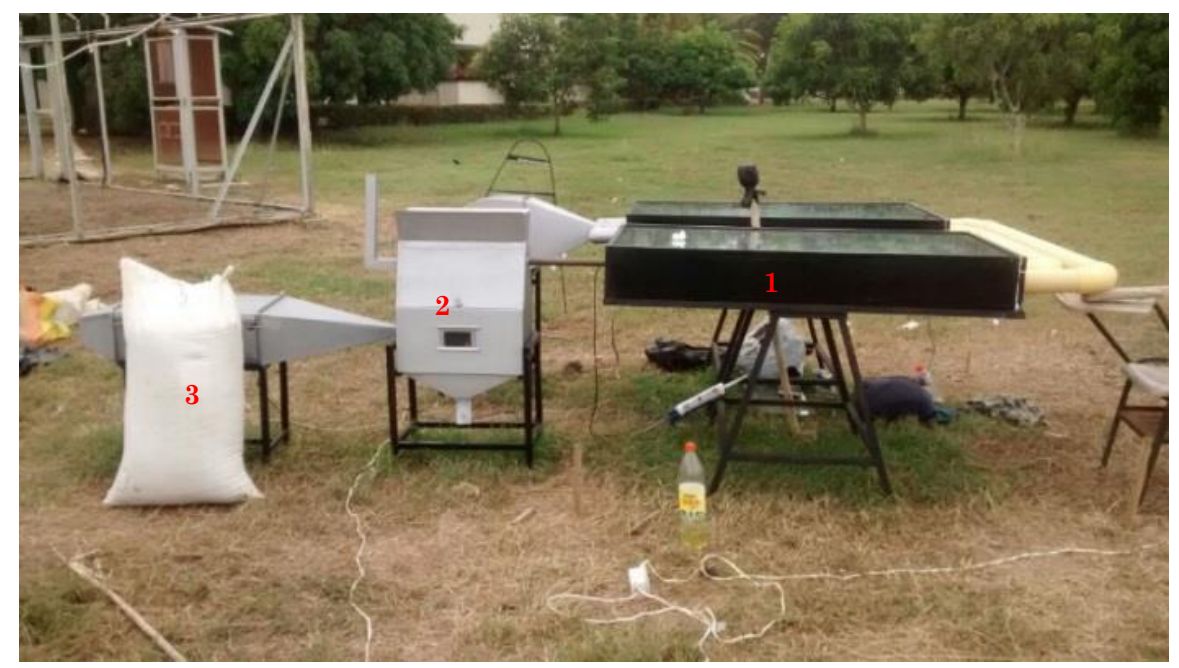

Fig. 9. Fotografía del secador híbrido compuesto de los colectores solares

(1), el quemador de biomasa (2 y el secador (3). Fuente: Autores.

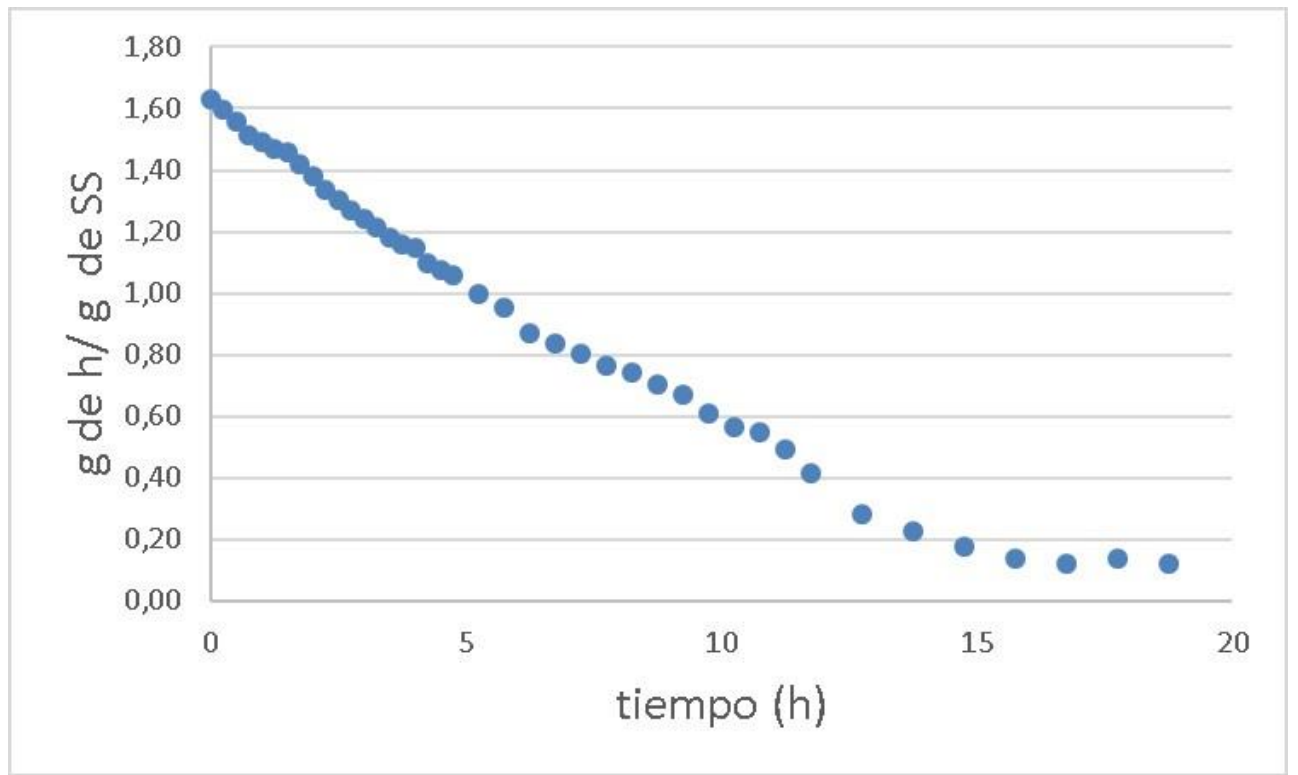

Fig. 10. Curva de secado a temperatura de $70^{\circ} \mathrm{C}$. Fuente: Autores.

\section{CONCLUSIONES}

En la simulación, la placa absorbedora fue el componente que mayor energía térmica le transfirió al aire durante el secado de la yuca, seguido de la parafina y la cubierta de vidrio.

El número óptimo de latas llenas de parafina para el banco de tubos en cada colector según la simulación del proceso térmico fue de 50 latas.

La disposición en serie de los colectores solares permitió aumentar de $5{ }^{\circ} \mathrm{C}$ la tem- peratura del aire de secado proveniente del primer colector.

El PCM permitió seguir el proceso de secado, incluso cuando la radiación solar estuvo por debajo de 116,22 $\pm 31,94 \mathrm{~W} / \mathrm{m} 2$, logrando mantener temperaturas de aire de secado en los dos colectores solares a 46 $\pm 4,29^{\circ} \mathrm{C}$ y $51 \pm 4,08^{\circ} \mathrm{C}$ durante $45 \mathrm{~min}$ adicionales.

En el secado de la yuca se necesitó $1 \mathrm{~h}$ y 33 min más, en comparación con el tiempo empleado para secarla en el laboratorio a $70^{\circ} \mathrm{C}$, presentando un periodo de velocidad 
Diseño y construcción de un sistema híbrido de calentamiento de aire por combustión de biomasa y radiación solar, utilizando PCM como fuente de almacenamiento térmico, para secado de yuca

de secado constante con una diferencia de 9 $\mathrm{h}$ y un periodo de velocidad de creciente 1 hora y 45 minutos, por encima del obtenido en el laboratorio.

\section{REFERENCIAS}

[1] G. V. B.-C. Albert Ibarz Ribas, Operaciones unitarias en la ingeniería de alimentos, 4ta ed. Mé. Madrid, Barcelona, México: Mundi Prensa, 2005.

[2] C. J. Geankoplis, Procesos de transporte y operaciones unitarias, 3rd ed. Ciudad de México: Compañía Editorial Continental, 1998.

[3] C. C. Bergues-Ricardo and J. R. Díaz-López., "Diagramas de tendencia para la generalización sostenible de secadores solares directos de productos agropecuarios," $R T Q$, vol. 34, no. 2, pp. 178-188, 2014.

[4] J. C. B. J. Alvaro Hernán Restrepo Victoria, "Disponibilidad térmica solar y su aplicación en el secado de granos," Sci. Tech., vol. 1, no. 27, pp. 127-132, 2005.

[5] A. G. M. B. Mustayen, S. Mekhilef, and R. Saidur, "Performance study of different solar dryers: A review," Renew. Sustain. Energy Rev., vol. 34, pp. 463-470, Jun. 2014.

[6] S. M. Shalaby, M. A. Bek, and A. A. ElSebaii, "Solar dryers with PCM as energy storage medium: A review," Renew. Sustain. Energy Rev., vol. 33, pp. 110-116, May 2014.

[7] J. Mumba, "Development of a photovoltaic powered forced circulation grain dryer for use in the tropics," Renew. Energy, vol. 6, no. 7, pp. 855-862, Oct. 1995.

[8] M. Condori, R. Echazu, G. Duran, and L. SARAVIA, "Secador Solar Híbrido. Diseño y construcción," Av. En energias Renov. $y$ Medio Ambient., vol. 12, p. 37, 2008.

[9] M. Condorí, R. Echazú, and L. Saravia, "Secador solar indirecto con flujo de aire forzado para huacalera, quebrada de humahuaca," Av. en Energías Renov. y Medio Ambient., vol. 10, pp. 47-54, 2006.

[10] R. A. A., J. A. Montoya, and C. A. Orozco., "Modelado dinámico de un secador híbrido para Guadua angustifolia Kunth," Sci. Tech., vol. 2, no. 42, pp. 345-350, 2009.

[11] J. J. F. Palacin, A. F. L. Filho, E. de C. Melo, and E. C. Teixeira, "Secagem combinada de café cereja descascado," CAPA, vol. 17 , no. 3 , pp. 244-258, 2009.

[12] M. Condorí, G. Durán, R. Echazú, and G. D. Russo, "Secador industrial híbrido solar biomasa para la producción continua de pimiento deshidratado," Energías Renov. y
Medio Ambient., vol. 25, pp. 81-92, 2010.

[13] P. L. T. K. Henry Rigit, Andrew Ragai, Jakhrani, Abdul Qayoom, Kamboh, Shakeel Ahmed, "Development of an Indirect Solar Dryer with Biomass Backup Burner for Drying Pepper Berries," World Appl. Sci. J., vol. 22, no. 9, p. 1241, 2013.

[14] Y. M. Yunus, H. H. Al-Kayiem, and K. A. K. Albaharin, "Design of a Biomass Burner/Gasto-gas Heat Exchanger for Thermal Backup of a Solar Dryer," J. Appl. Sci., vol. 11, no. 11, pp. 1929-1936, Nov. 2011.

[15] P. T. E. Tarigan, "A Mixed-Mode Natural Convection Solar Dryer with Biomass Burner and Heat Storage Back-up Heater," Hat Yai, Songkhla, Thailand, 2005.

[16] J. Prasad, V. K. Vijay, G. N. Tiwari, and V. P. S. Sorayan, "Study on performance evaluation of hybrid drier for turmeric (Curcuma longa L.) drying at village scale," J. Food Eng., vol. 75, no. 4, pp. 497-502, Aug. 2006.

[17] J. Prasad, V. K. Vijay, G. N. Tiwari, and V. P. S. Sorayan, "Study on performance evaluation of hybrid drier for turmeric (Curcuma longa L.) drying at village scale," J. Food Eng., vol. 75, no. 4, pp. 497-502, Aug. 2006.

[18] S. Devahastin and S. Pitaksuriyarat, "Use of latent heat storage to conserve energy during drying and its effect on drying kinetics of a food product," Appl. Therm. Eng., vol. 26, no. 14-15, pp. 1705-1713, Oct. 2006.

[19] IDAE, "Biomasa - IDAE, Instituto para la Diversificacion y Ahorro de la Energía." [Online]. Available: http://www.idae.es/index.php/idpag.895/relm enu.321/mod.pags/mem.detalle. [Accessed: 25-Mar-2017].

[20] A.O.A.C., "Official Methods of Analysis of AOAC International," Assoc. Off. Anal. Chem. Int., p. Method ce 2--66, 2000.

[21] J. L. M. E. e I. I. M. H. Everaldo Joaquín Montes Montes, Ramiro Torres Gallo, Ricardo David Andrade Pizarro, Omar Andrés Pérez Sierra, "Modelling the kinetics of thin-layer yam (dioscorea rotundata) drying," Ing. Investig., vol. 28, no. 2, pp. 45$52,2008$.

[22] A. Sharma, V. V. Tyagi, C. R. Chen, and D. Buddhi, "Review on thermal energy storage with phase change materials and applications," Renew. Sustain. Energy Rev., vol. 13, no. 2, pp. 318-345, Feb. 2009.

[23] L. M. Bal, S. Satya, S. N. Naik, and V. Meda, "Review of solar dryers with latent heat storage systems for agricultural products," Renew. Sustain. Energy Rev., vol. 15, no. 1, pp. 876-880, Jan. 2011. 
Diseño y construcción de un sistema híbrido de calentamiento de aire por combustión de biomasa y radiación solar, utilizando PCM como fuente de almacenamiento térmico, para secado de yuca

[24] A. Prada and C. E. Cortés, "Thermal decomposition of rice husk: an alternative integral use," Orinoquia, vol. 14, no. 1, pp. 155-170, 2010.

[25] P. Gbaha, H. Yobouet Andoh, J. Kouassi Saraka, B. Kaménan Koua, and S. Touré, "Experimental investigation of a solar dryer with natural convective heat flow," Renew. Energy, vol. 32, no. 11, pp. 1817-1829, Sep. 2007.

[26] A. Saleh and I. Badran, "Modeling and experimental studies on a domestic solar dryer," Renew. Energy, vol. 34, no. 10, pp.
2239-2245, Oct. 2009.

[27] J. S. Mendoza, J. L. M. B., M. V. B., A. F. Q., and M. L. V. M., "Cinética de secado de la yuca (Manihot esculenta Crantz) variedad CORPOICA M-tai en función de la temperatura y de la velocidad de aire," Rev. ion, vol. 27, no. 2, pp. 29-42, 2014.

[28] W. T. de Carvalho, T. F. de Oliveira, F. A. da Silva, M. Caliari, and M. S. Soares Júnior, "Drying kinetics of potato pulp waste," Food Sci. Technol., vol. 34, no. 1, pp. 116-122, Mar. 2014. 